transmitted infection (STI) rates among young adults. This study examines whether contextual factors affect young adults' perceived STI risk and engagement in sexual risk behaviours. In addition, we compare sexual histories among app users and non-users.

Methods We recruited our sample from 111 college Facebook groups over a 10 week period. Participants were presented with 1 of 32 scenarios varying in levels of perceived risk. They answered questions pertaining to relationship status, dating app and sexual experience, condom usage, and STI testing experience.

Results A total of 4429 eligible participants between the ages of 18-24 completed the survey. Participants were more likely to believe that their partner had a greater number of sexual partners and engaged in casual sex if the scenario involved either a male partner, perceived high-risk location, or onenight stand. They were more likely to enforce condom usage if their partner was male. Lastly, they were more likely to ask about their partner's STI status if the scenario involved a male partner or one-month dating. Among sexually active participants, dating app usage was associated with sexual experience and having casual relationships, more sexual partners, higher perceived STI risk, and STI testing. While over half of the sexually active participants had inconsistent condom usage and had not been tested for STIs, they generally reported low perceived STI risk.

Conclusion Dating app users are more likely to engage in sexual risk behaviours and to have had STI testing. For these reasons, dating apps can be a useful platform for increasing STI knowledge and reducing the incidence of STIs among their users.

\section{P4.25 IMPROVING EFFICACY OF THE HIV RESPONSE THROUGH INTEGRATED FAMILY SERVICES: A MICRO- SIMULATION}

Chris Desmond, Furzana Timol. Human Sciences Research Council, Durban, South African Republic

\subsection{6/sextrans-2017-053264.522}

Introduction With antiretroviral treatment more commonly available there is a gradual shift in the response to HIV from the emergency response to the management of a chronic condition. This requires that we shift our approach not only to treatment, but also to prevention and the protection of affected children. We examine through micro-simulation modelling the potential benefits of more highly integrated responses.

Methods Using South African data we undertake household level micro-simulation modelling examining the dynamic relationship between adult incidence, access to treatment and treatment adherence, and the long term impact on children, including in ways which increase their risk of HIV infection as adults. We simulate a cohort of women born in 1985 examining the consequences of the South African context for their and their children's survival and their children's HIV risk profile.

Results In the South African context over 35\% of children will be affected by maternal HIV. This leads to lower rates of school completion (12 percentage points) and higher rates of adolescent mental health issues (10 percentage points). Both of these outcomes have been linked to HIV risk behaviours.
The results are highly sensitive to adult treatment uptake and adherence, as both affect the timing of adult illness and death.

Conclusion The study highlights the links between adult and child outcomes, not only in terms of negative developmental outcomes for children, but in terms of the epidemics replications. This suggests the need to, when appropriate, treat the family as a unit and focus on addressing common household challenges to adult adherence and adolescent risk behaviour. The results suggest that the current silo approach, common in much of Africa, is economically inefficient.

Funding for this study was provided by USAID through Management Sciences for Health.

\section{P4.26 COULD DIFFERENCES IN IMPLICIT ATTITUDES TO SEXUAL CONCURRENCY PLAY A ROLE IN GENERALISED HIV EPIDEMICS?}

${ }^{1}$ Chris Kenyon, ${ }^{1}$ Kara Osbak, ${ }^{2}$ Kenny Wolfs, ${ }^{3}$ Maleeto Malataliana, ${ }^{3}$ Sizwe Zondo, ${ }^{4}$ Guido Van Hal, ${ }^{2}$ Jacques Van Lankveld. ${ }^{1}$ Institute of Tropical Medicine, Antwerp, Belgium; ${ }^{2}$ Open University, The Netherlands, Heerlen, The Netherlands; ${ }^{3}$ Rhodes University, South Africa, Grahamstown, South African Republic; ${ }^{4}$ Antwerp University, Belgium, Antwerp, Belgium

\subsection{6/sextrans-2017-053264.523}

Introduction High rates of sexual partner concurrency have been shown to facilitate the spread of various sexually transmitted infections. Assessments of explicit attitudes to concurrency have however found little difference between populations. We developed a concurrency implicit associations test (C-IAT) to assess if implicit attitudes towards concurrency may vary between individuals and populations and what the correlates of these variations are.

Methods We recruited 869 Belgian (mean age 22.9, SD 5.1) and 70 South African (mean age 22.1, SD 2.5) university students to complete the C-IAT together with a questionnaire concerning sexual behaviour and explicit attitudes to concurrency.

Results The Belgian students C-IATs demonstrated a strong preference for monogamy $(-0.78, \mathrm{SD}=0.41)$, with $93.2 \%$ of participants having a pro-monogamy C-IAT. The South Africans' C-IAT demonstrated little preference for concurrency or monogamy $(-0.009, \mathrm{SD}=0.43)$, with $34.7 \%$ having a promonogamy C-IAT $(\mathrm{p}<0.0001)$. The South Africans also reported more concurrent sexual behaviour than the Belgians. At a population- but not an individual-level, the C-IAT was a better predictor of actual concurrent behaviour than explicit norms towards concurrency.

Conclusion We found larger differences in implicit than explicit attitudes towards concurrency between populations. These findings need to be replicated in larger samples.

\section{P4.27 HEALTHCARE ACCESS AND ANTIBIOTIC USE FOR GENITOURINARY SYMPTOMS AMONG FEMALE SEX WORKERS IN TIJUANA MEXICO}

${ }^{1}$ Claire C Bristow, ${ }^{2}$ Jeffrey D Klausner, ${ }^{1}$ Sheldon R Morris, ${ }^{3}$ Alicia Vera, ${ }^{4}$ Ac Vargas-Ojeda, ${ }^{1}$ Heather A Pines, ${ }^{1}$ Shirley J Semple, ${ }^{1}$ Thomas L Patterson. 'University of California San Diego, La Jolla, USA; ${ }^{2}$ University of California Los Angeles, Los Angeles, USA; ${ }^{3}$ U.S. Mexico Border Health Commission, Tijuana, Mexico; ${ }^{4}$ Universidad Autonoma De Baja California, Mexico, Tijuana, Mexico

10.1136/sextrans-2017-053264.524 
Introduction Female sex workers (FSW) may experience barriers to accessing sexual health services. Appropriate treatment for STIs is paramount to prevent the spread of antibiotic resistant infections. We aimed to understand how healthcare access might impact self-treatment and antibiotic use for genitourinary symptoms among FSWs in Tijuana, Mexico.

Methods 282 FSW participants were tested for STIs and underwent an interview. APTIMA COMBO 2 (Hologic, Inc.) was used to diagnose cervicovaginal Neisseria gonorrhoeae (NG) infection. We summarised data and used logistic regression to assess associations between self-medication (use of pills and/or injections that were not prescribed by a doctor or nurse) for genitourinary symptoms and prior HIV test (a proxy for accessing sexual health services), income and STI history. Genitourinary symptoms included dysuria, vaginal discharge, vaginal bleeding between periods, pelvic pain, or dyspareunia.

Results 282 FSW participants were recruited in Tijuana. Of the 263 with test results, $41(15.6 \%)$ tested positive for NG, of which $23(56.1 \%)$ endorsed current genitourinary symptoms. Of the 282 total participants, $60(21.3 \%)$ reported that they had ever self-medicated to treat genitourinary symptoms. Never having an HIV test $(\mathrm{OR}=2.3$ (95\% CI: 1.3, 4.3)) and reporting lower income $(<\mathrm{MX} \$ 3500$ vs. $\geq \mathrm{MX} \$ 3500)$ $(\mathrm{OR}=2.2 \quad(95 \% \mathrm{CI}: 1.1,3.6)$ were associated with reporting ever self-medicating for treatment of genitourinary symptoms. In addition, those that reported they had ever had an STI $(\mathrm{n}=83 \quad(29.4 \%), \quad \mathrm{OR}=2.7 \quad(95 \% \mathrm{CI}: 1.5,4.8))$ were more likely to report self-medication for treatment of genitourinary symptoms.

Conclusion We found a high prevalence of NG infection among a cohort of FSWs in Tijuana, Mexico. The high prevalence and past medication use without a prescription point to a potential for emerging NG antibiotic resistance in this setting. Barriers to access for sexual healthcare may drive the use of medications without a prescription. Additionally, those with a history of STIs may be self-treating for familiar symptoms.

\section{P4.28 STIGMA AND DISCRIMINATION EXPERIENCES IN HEALTH CARE SETTINGS MORE EVIDENT AMONG TRANSGENDER PEOPLE THAN MALES HAVING SEX WITH MALES (MSM) IN INDONESIA, MALAYSIA, THE PHILIPPINES AND TIMOR LESTE: KEY RESULTS}

RN Cortes. The Philippine Ngo Council on Population, Health and Welfare, INC., Pasay City, Philippines

\subsection{6/sextrans-2017-053264.525}

Introduction A four-country study was conducted by the ISEAN-Hivos Program (Global Fund regional AIDS grant) which aims to provide information on the status of stigma and discrimination (SAD) among males having sex with males (MSM) and transgender people in health care settings (including HIV/AIDS services) using a questionnaire based on the forms of SAD described in the Stigma and Discrimination Index Questionnaire.

Methods The study's questionnaire described SAD in terms of the respondents' self-reported perception of: 1 . Refusal of health care services, 2. Physical maltreatment, 3. Verbal maltreatment, and 4. Provision of health care service below standards. A total of 2409 respondents, 30\% $(n=719)$ of whom are self-identifying male-to-female trans persons, participated in this study. There were 264 trans respondents from Indonesia, 204 in the Philippines, 174 in Malaysia, and 77 in Timor Leste.

Results Overall, the results indicate that significantly more trans people experienced SAD in health care settings compared to MSM. Verbal maltreatment was the most commonly experienced $(24.26 \%)$, followed by receiving a perceived low quality of health service (22.57\%), being refused access to health care services $(18.23 \%)$ and lastly, physical maltreatment (18.21\%). There were proportionately more SAD experiences reported by trans people in Timor Leste (41.06\%), followed by Malaysia (32.67\%), Philippines (7.47\%) and Indonesia $(2.0 \%)$.

Conclusion The result of the SADS suggests that there is a wide variation across the four countries in terms of trans people experiencing stigma and discrimination. Verbal maltreatment is the topmost common form of SAD among trans people. The study indicates that trans' personal experiences of SAD are more frequent than MSM. Also, about one third of the trans people continue to experience SAD. Almost $60 \%$ of the trans people, however, did nothing to address SAD. SAD still exists in health care settings, which needs support for more interventions to significantly decrease, if not totally eradicate SAD in its many forms.

\section{P4.29 THE VALUE OF BUILDING SOCIAL NETWORKS AMONG NIGERIAN MEN WHO HAVE SEX WITH MEN AFFECTED BY SEXUAL STIGMA: A QUALITATIVE STUDY OF BARRIERS AND BENEFITS}

${ }^{1}$ Cristina Rodriguez-Hart, ${ }^{2}$ Cory Bradley, ${ }^{2}$ Danielle German, ${ }^{3}$ Ifeanyi Orazulike, ${ }^{4}$ Blessing Kayode, ${ }^{5}$ Hongjie Liu, ${ }^{6}$ Trevor A Crowell, ${ }^{2}$ Stefan Baral, ${ }^{1}$ Man Charurat, ${ }^{1}$ Rebecca $G$ Nowak. 'Institute of Human Virology University of Maryland School of Medicine, Baltimore, USA; ${ }^{2}$ Johns Hopkins Bloomberg School of Public Health, Baltimore, USA ${ }^{3}$ International Centre on Advocacy and Rights to Health, Abuja, Nigeria; Institute of Human Virology Nigeria, Abuja, Nigeria; ${ }^{5}$ University of Maryland School of Public Health, College Park, USA; ${ }^{6}$ U.S. Military Hiv Research Program, Walter Reed Army Institute of Research, Silver Spring; Henry, Bethesda, USA

\subsection{6/sextrans-2017-053264.526}

Introduction Sexual stigma affecting men who have sex with men (MSM) in Nigeria may contribute to acquisition and onward transmission of HIV and other sexually transmitted infections (STIs). Prior analysis found stigma to be associated with increased HIV and STI prevalence and larger MSM social network size. The aim of this study was to explore the association between stigma and MSM social networks.

Methods From March 2013 to February 2016, the TRUST/ RV368 study recruited 1,480 MSM in Abuja and Lagos, Nigeria into a prospective cohort that provides HIV and STI diagnosis and treatment. From this parent study, 15 semistructured in-depth interviews were conducted with participants who experienced elevated stigma. Interviews were transcribed and coded using thematic analysis.

Results The key themes for how sexual stigma may have led to greater vulnerability for HIV and STIs were: financial vulnerability and emotional trauma, constrained opportunities to share HIV and STI resources, and internet use. Participants described financial exploitation and emotional trauma as common consequences of stigma that at times was associated with condomless sex and transactional sex. Fear of stigma constrained MSM's willingness to openly socialise, which may have limited exposure to sexual health services, as MSM social 
networks were often described as a medium for emotional support, information on HIV/STIs, and referrals to clinics serving MSM. Instead, many relied on the internet to find MSM. This strategy was considered to be safer, but it may have increased participants' exposure to HIV/STIs through exposure to more sex partners and increased their vulnerability for blackmailers to locate and extort them for money.

Conclusion These interviews suggest that interventions that reduce sexual stigma and leverage social resources in networks among Nigerian MSM can support improved HIV and STI outcomes such as improved awareness of services, earlier diagnosis and engagement in care. Capitalising on the broad use of social media applications represents an important platform for these services.

\section{P4.30 INTEGRATING SEXUAL AND REPRODUCTIVE HEALTH EDUCATION IN PREVENTING THE SPREAD OF STI \& AIDS}

Danielle Guerrero Saique.

10.1136/sextrans-2017-053264.527

Cswdo, Akap-Bata, Dasmarinas - Philippines

Introduction Sexual and Reproductive Health (SRH) encompasses all stages of life. Women have rights to quality life. It is attained by practicing, exercising SRH in preventing Violence Against Women (VAW). SRH education is integrated in the total health package service-delivery system of the Medical Social Worker (MSWr) and health service-providers availed from the medical-health services, from health facilities. This paper presented the cause, prevalence, effects of VAW for not practicing/violating SRH. It proposed to the Stakeholders to formulate Matrix of Action Plan by integrating SRH in the holistic approach of the Medical Social Work, practice and health service-delivery thereby preventing VAW and spread of STI and AIDS.

Method VAW cases showed that victim and violators are known to each other, related, living together. Cases of VAW transpired at home, reported, investigated in the police station, filed in the legal courts of law inclusive of Y-2016 from the City of Dasmariñas. Data from police blotters, reports, filed cases, social/medical case studies were gathered by MSWr. Qualitative analysis identified causes, prevalence, effects of VAW related in violating SRH. The MSWr served innovative interventions by implementing his/her SRH background, knowledge/skills in educating/counselling and managing cases of the client-victims to end VAW and prevent spread of STI and AIDS.

Results 65 VAW cases reported the causes and effects related to violating SRH were varied from: Due to non-negotiation or refusal of practicing SRH by the couples, non-acceptance of Family Planning, wrong choice of FP methods by the partners yielded helpless battered women, transmission of STI and AIDS, unwanted and unplanned pregnancies, abandoned and neglected children. Unattended or neglected pre-post natal/ maternal care caused medical complications and death of mother or infant. Rape, incest led to adverse trauma effect to the changing behaviour of the victim and result to her suicide or death. Unsafe, unprotected sex transmitted STD and AIDS to the incest or rape victim. From the presented cases, VAW is eliminated by promoting the $\mathrm{SRH}$ and preventing the spread of STI and AIDS.
Conclusion Youths, PWDs, LGBTs, Elders of non-health seeking-behaviour, non-access to SRH from health facilities, from MSWr, health service-providers violated the: Rights to life, quality health care/protection; Rights to information/education; Rights to plan family; Rights from torture/ill-treatment. VAW brings adverse effects of psychosocial trauma to victim's wellness/well-being and humaneness. MSWr's interventions on SRH and VAW's findings/results are recommendations to Stakeholders in making quality SRH education available by integrating SRH to the health-service delivery thereby promoting SRH: empowering women's rights to development, gender equality, thus preventing the spread of STI and AIDS and ending VAW. Therefore, Stakeholders must vouch in duplicating this best-practice to access SRH as implemented by the MSWr and health service-providers from the medical-health facilities.

\section{P4.31 KISSING IS ASSOCIATED WITH THE SOURCE FOR MEETING CASUAL PARTNERS: AN IMPLICATION FOR GONORRHOEA TRANSMISSION AND CONTROL IN MEN WHO HAVE SEX WITH MEN}

David Priest, Christopher K Fairley, Sandra Walker, Vincent J Cornelisse, Marcus Chen, Catriona S Bradshaw, Tiffany Phillips, Eric P.F. Chow. Melbourne Sexual Health Centre, Melbourne, Australia

\section{$10.1136 /$ sextrans-2017-053264.528}

Introduction Kissing has been identified as one of the most common sexual practices among men who have sex with men (MSM), and it has also been identified as one of the risk factors for pharyngeal gonorrhoea. We conducted this study to understand the kissing pattern amongst MSM by different sources for meeting partners.

Methods A cross-sectional questionnaire-based study was conducted amongst MSM attending Melbourne Sexual Health Centre between March and September 2015. Participants were asked about the sources they used to meet their casual sexual partners and about their kissing practices in the previous three months. Multivariate logistic regression analyses were performed to determine whether kissing is associated with the source for meeting partners.

Results A total of 753 men completed the questionnaire with a median age of 29 (IQR 25-36). Our results showed that men who met partners at gay bars were 7.3 (95\% CI 2.125.0) times more likely to kiss their sexual partners and they were less likely (aOR 0.6; 95\% CI 0.4-0.8) to have sex-without-kissing partners after adjusting for age and other sources for meeting partners. Men who met partners via smartphone applications were 7.0 (95\% CI 3.0-15.9) times more likely to kiss their partners. Kissing was not associated with men who met partners at sex on premises venues (SOPV) and via friends. In contrast, men who met partners at SOPV were 2.3 (95\% CI 1.6-3.3) times more likely to have sex-without-kissing partners.

Conclusion There is considerable difference in kissing practices among men who met partners at different locations. Our data suggest kissing may be a more important contributor to gonorrhoea transmission among men who met partners at gay bars, while penile-anal sex may be the major mode of gonorrhoea transmission among men who met partners at SOPV. 


\section{P4.32 HOMOSEXUAL TO BISEXUAL: OVERLAPPING SEXUAL RISKS AMONG MEN WHO HAVE SEX WITH MEN AS WELL AS WOMEN IN SOUTH ASIAN COUNTRIES}

Deepanjali Vishwakarma. International Institute for Population Sciences, Mumbai, India

10.1136/sextrans-2017-053264.529

Introduction Heterosexual relations among bisexual MSM engender spectrum of vulnerabilities for MSM as well as their partners. Socio-cultural pressure to marry may drive MSM to have female partners, indicating the potential for bridging HIV transmission. However, these interactions are poorly understood, and measurement of potential risk to MSM and their female partners is inconsistent in most of the South Asian countries. The study aims to analyse the overlapping male and female partnerships of MSM-SW given the multiplicity of risk for HIV.

Methods This paper uses data collected as a part of mapping and size estimation of MSM in Nepal, Bhutan, and Bangladesh and for India, the IBBA has been used (2010-2016). Analytical methods range from cross-country comparisons of tangible indicators to application of regression.

Results Bisexuality in the region is rampant as a substantial proportion of MSMs ( $42 \%$ in India to $74 \%$ in Bhutan) who have sex with a male in the last 6 months also reported to sleeping with a female partner during the same period. MSM age 20-29 years, who are never married and migrants are significantly more likely to have multiple female partners in each of the 4 countries. However, the considerably lower prevalence of condom use, among those having sex with multiple female partners in the last six months across the countries is a marker of the multiplicity of the STIs/HIV risk. Migration is playing an inverse role in determining the relationship with condom use in the last sex with female partner as those MSM who are non-migrants were more likely to use condom. Conclusion Role of MSM-SW in HIV transmission is a more complex issue than depictions of men as sexual predators and women as uninformed victims. MSM programs should envisage beyond homosexuality and address bisexuality regardless of their sexual identity as threat clouding prevention efforts by increasing the thrust of programs designed for MSMs.

\section{P4.33 A RELATIONSHIP BASED SEXUAL HEALTH FRAMEWORK FOR STI PREVENTION AMONG ADULT STI CLINIC ATTENDEES}

J Devon. J Hensel;. Dennis Fortenberry. Indiana University School of Medicine, Indianapolis, USA

10.1136/sextrans-2017-053264.530

Introduction Relationship-based sexual behaviours are important elements in sexual health approaches to STI prevention.

Method STI-clinic recruited men and women $(n=423,18-$ 30 years.) contributed quarterly sexual health information and weekly urine-based STI specimens over 12 weeks. Sexual health was a standardised, 10-item index of partner-associated measures (relationship quality, sexual satisfaction, control over sexual activity, shared social and sexual decision making, condom use efficacy, emotional commitment, partner meeting needs, knowing partner well, frequency of seeing partner, perceived STI chances). STI- behaviours were: condom use during vaginal sex, condom use during anal sex, given money for sex (no/yes) and partner forced sex (no/yes). STI was NAAT diagnosis of chlamydia (CT), gonorrhoea (GC) and trichomonas (TV). Analyses were mixed-effect logistic or poisson regression (Stata, v.13); models were stratified by gender and monogamy, controlling for age, race and partner age difference, and number of partners and condom use (STI models).

Results Sexual health was associated with more frequent condom use during vaginal sex for men and women $(\mathrm{IRR}=1.92-$ 2.18) and for monogamous and non-monogamous $(I R R=1.72-3.01)$ relationships, with lower odds of men's or women's $(\mathrm{OR}=0.16-0.19)$ being paid for sex, with lower odds of women's $(\mathrm{OR}=0.09)$ and monogamous and nonmonogamous relationships' $(\mathrm{OR}=0.24-0.15)$ reporting forced sex. Controlling for total partners and condom use, sexual health lowered men's and women' odds of CT $(\mathrm{OR}=0.38$ $0.43)$, men's $(\mathrm{OR}=0.40)$ and non-monogamous relationships' odds of GC $(\mathrm{OR}=0.41)$, and men's odds of TV $(\mathrm{OR}=0.33)$.

Conclusion Among men and women, and even in non-monogamous partnerships, a relationship-based measure of sexual health is linked to fewer STI risk behaviours and lower STI incidence. Our data are empirical evidence of the importance of sexual health in relationships as a means of STI control.

\section{P4.34 HIV PREVENTION IN ADOLESCENTS AND YOUNG PEOPLE: A SYSTEMATIC REVIEW}

Diego Agostinho Calixto. Ministry of Health of Brazil - Department of STI, HIVIAIDS and Viral Hapatitis, Brasilia, Brazil

\subsection{6/sextrans-2017-053264.531}

Introduction Systematically review and update evidence on the effectiveness of HIV prevention interventions and make recommendations for evidence-based interventions to guide efforts to meet the goals of the Special Session on Ending AIDS at the United Nations General Assembly in 2016 . HLM2016. In the HLM 2016 political declaration, grave concern is expressed that adolescents and young people aged 1524 represent more than a third of all new HIV infections among adults, with 2,000 HIV-infected young people per day. Methods Recent evidence from the last 5 years (January 2011 to December 2016) on the effectiveness of prevention interventions in young people to reduce HIV risk behaviour and their outcomes and impacts among young people was evaluated using PubMed.

Results A total of 23 studies were included after screening about 413 citations. Community-based and youth-led interventions focused on peer dialogue have demonstrated clear evidence of a reduction in reported sexual risk behaviours. Interventions in health services have increased the use of services when they are accessible and more favourable to young people. In geographically defined communities, communitycentred interventions and interventions have reduced reporting of at-risk sexual behaviours.

Conclusion HIV prevention among young people is a priority for the world. The most promising interventions need to be expanded now, with careful evaluation, exploring peer dialogue interventions to impact the incidence of HIV among adolescents and young people, especially young people and adolescents from key populations. 